\title{
DIARRÉIA AGUDA
}

\author{
ACUTE DIARRHEA
}

Ricardo B. de Oliveira

Docente.Divisão de Gastroenterologia. Departamento de Clínica Médica. Faculdade de Medicina de Ribeirão Preto - USP.

Correspondência: Prof. Dr. Ricardo B. de Oliveira. Departamento de Clínica Médica. Hospital das Clínicas - Campus Universitário CEP 14048-800 - Ribeirão Preto - SP. Tel: (16) 6022457.

OLIVEIRA RB. Diarréia aguda. Medicina, Ribeirão Preto, 36: 257-260, abr./dez. 2003.

RESUMO - A abordagem atual do paciente com diarréia aguda é apresentada.

UNITERMOS - Diarréia Aguda. Condutas Terapêuticas.

\section{GENERALIDADES}

A cada ano, um grande número de pessoas, no mundo inteiro, apresenta, pelo menos, um episódio agudo de diarréia. A maioria desses episódios causa desconforto tolerável e se resolve espontaneamente, em horas ou poucos dias, sem tratamento; quando a diarréia é intensa, ou acompanhada de sangramento, dor abdominal, febre ou outros sintomas, a busca de atenção do médico é a regra. Este capítulo apresenta uma abordagem prática das diarréias agudas.

Diarréia resulta de anormalidades funcionais do tubo digestivo,que produzam redução da absorção ou aumento da secreção de água e eletrólitos .

Secreção anormal - Diversos microorganismos (Vibrião da cólera, cepas de Escherichia coli, espécies de Salmonella, Campylobacter jejuni, etc) colonizam a luz intestinal e produzem toxinas capazes de ativar mecanismos de estímulo à secreção, existentes na mucosa intestinal (ativação da adenilatociclase ou da guanilatociclase, alterações das tight junctions intercelulares, liberação de serotonina pelas células enterocromafins do intestino). Nesses casos, há pouca ou nenhuma inflamação e é mínima a lesão direta ao epitélio intestinal, o qual é colocado em funcionamento inapropriado.

\begin{abstract}
Absorção defeituosa e perda anormal - Ocorre, quando há dano ao epitélio intestinal. Esse dano pode ser limitado à membrana das microvilosidades do epitélio ou ser extenso, com a descamação do epitélio e formação de úlceras, como as que decorrem da intensa inflamação em resposta a microorganismos invasivos (espécies de Shigella, por exemplo). Muitas vezes, além de má-absorção, há exsudação de plasma, sangue e fluidos inflamatórios, que contribuem para a diarréia.
\end{abstract}

Diarréia osmótica - Defeitos da absorção de nutrientes no intestino delgado, assim como ingestão de substância inabsorvíveis ( laxativos, fenformina ) resultam em resíduos de osmolaridade elevada na luz dos cólons, que impedem a absorção de água, ainda que o epitélio se encontre normal .

Motilidade anormal - Grandes volumes intraluminares, resultantes dos mecanismos antes expostos, estimulam, reflexamente, a motilidade intestinal, tornando o trânsito intestinal rápido, o que contribui para a diarréia. Toxinas ou fármacos (eritromicina, por exemplo) que determinam a instalação de padrões de motilidade propulsivos têm o mesmo efeito.

Mecanismos combinados - Quando há inflamação na parede intestinal, tanto a secreção de eletrólitos e de muco, como a motilidade propulsiva, é 
estimulada por mecanismos que envolvem a ação direta, bem como a intermediada pelo sistema nervoso entérico de mediadores de inflamação sobre as glândulas e a musculatura lisa do intestino.

\section{DIAGNÓSTICO}

O diagnóstico de diarréia não apresenta, em geral, dificuldades: o próprio paciente o firma corretamente. Ao médico caberá:

1. confirmar o diagnóstico, certificando-se de que a diarréia relatada é real, e não apenas a passagem freqüente de fezes formadas;

2. avaliar a gravidade, com atenção para:

- volume das perdas;

- estado de hidratação;

- presença de febre;

- presença de hematoquesia ( sangue nas fezes); - presença e características de dor abdominal; - envolvimento simultâneo de outros órgãos ou sistemas;

3. esclarecer se o comprometimento é de segmentos distais (evacuações são muito freqüentes, com sangue vivo, urgência ou tenesmo) ou proximais (evacuações em pequeno número, volumosas) do tubo digestivo;

4. obter evidência sobre a etiopatogenia (infecciosa, por toxina pré-formada, iatrogênica, etc). A Tabela I apresenta causas comuns de diarréia iatrogênica.

A Tabela II apresenta os principais agentes infecciosos, que causam diarréia aguda.

Tabela I - Medicações de uso comum com potencial de causar diarréia

- Laxativos

- Antibióticos

- Procinéticos

- Fenformina

- Diuréticos

- Alopurinol, colchicina

- Teofilina

- Antiinflamatórios
Tabela II - Agentes infecciosos que causam diarréia aguda

\section{Agente}

\section{BACTÉRIAS}

- Vibriões (cólera e outros)

- Escherichia coli enterotoxigênica

- Escherichia coli enteropatogênica

- Escherichia coli entero hemorrágica*

- Escherichia coli entero"agregativa"

- Escherichia coli enteroinvasiva *

- Shigella SPP*

- Salmonella SPP*

- Campylobacter SPP*

- Yersinia enterocolitica

- Clostridium difficile*

- Micobacteria*

\section{METAZOÁRIOS}

- Strongyloides stercoralis

- Schistosoma SPP*

PROTOZOÁRIOS

- Giárdia intestinalis

- Criptosporidium parvum

- Microsporídia

- Isospora belli

- Cyclospora cayetanensis

- Entamoeba hystolitica*

- Balantidium coli

VÍRUS

- Rotavírus

- Adenovírus

- Calicivírus

- Astrovírus

- Citomegalovírus*

* Causa de enterorragia 


\section{CONDUTAS}

As seguintes informações são relevantes para a definição de condutas em pacientes com diarréia aguda:

1. a maioria dos episódios de diarréia aguda é autolimitado;

2. a cultura de fezes é positiva em uma minoria de episódios;

3. a maioria das cepas de Escherichia coli, Salmonela e Shigella é sensível às quinolonas;

4. a probabilidade de infecção por Shigella é expressiva, quando há hematoquesia;

5. em indivíduos imunocompetentes, a evolução do quadro clínico de Shigellose, mas não de Salmonellose, é influenciada, favoravelmente, pela administração de antimicrobianos;

6. o risco para a síndrome hemolítico/urêmica é elevado, quando se administram antibióticos a portadores de Escherichia coli $\mathrm{O} 157 \mathrm{H} 7$ (portadora do bacilo de Shiga);

7. o uso de antibióticos contribui para o desenvolvimento de cepas de microorganismos resistentes, implica em risco de iatrogenia e de colite pseudomembranosa;

8. as manifestações da infecção pelo Campylobacter jejuni são abreviadas pela administração de eritromicina.

\section{A - Indivíduos previamente hígidos}

1) Temperatura $<38^{\circ} \mathrm{C}$, sem hematoquesia, manifestações sistêmicas ou sinais de desidratação ausentes

- Restringir a atividade física.

- Manter a hidratação, por via oral - reduzir a ingestão de alimentos e aumentar a de líquidos.

- Considerar a administração judiciosa de loperamida ou difenoxilato, prescrevendo doses individuais, conforme a necessidade.

- Administrar antimicrobianos no contexto de epidemia ou surto por microorganismo conhecido, para o qual haja tratamento de eficiência comprovada.

- Coletar fezes para cultura, se quadro durar mais de $48 \mathrm{~h}$.

2) Temperatura $>38{ }^{\circ} \mathrm{C}$, sem hematoquesia, manifestações sistêmicas ou sinais de desidratação ausentes

- Restringir a atividade física.

- Manter a hidratação pela via oral; reduzir a ingestão de alimentos e aumentar a de líquidos.

- Considerar a administração judiciosa de loperamida ou difenoxilato, prescrevendo doses individuais, conforme a necessidade.

- Coleta de fezes para cultura e parasitológicos.

- Manter o paciente sob observação; administrar antimicrobianos, se febre perdurar por mais de 48 $\mathrm{h}$, se manifestações sistêmicas aparecerem ou se houver a suspeita forte de infecção por microorganismo para o qual haja tratamento com eficiência comprovada (p. ex. Shigella SP).

3) Com hematoquesia, manifestações sistêmicas ou sinais de desidratação ausentes

- Restringir a atividade física.

- Manter a hidratação, com preferência pela via oral; reduzir a ingestão de alimentos, e aumentar a de líquidos.

- Não administrar loperamida ou difenoxilato, se houver suspeita de infecção por Escherichia coli, portadora da toxina de Shiga (STEC).

- Avaliar a necessidade de transfusão de sangue.

- Considerar diagnóstico diferencial com as causas de hemorragia digestiva baixa.

- Internar e realizar endoscopia imediatamente, se o volume de sangue perdido for expressivo.

- Administrar antimicrobiano, apenas se houver suspeita forte de infecção por microorganismo, para o qual haja tratamento com eficiência comprovada ( p. ex., Shigella).

4) Temperatura $>38{ }^{\circ} \mathrm{C}$, com manifestações sistêmicas expressivas

- Restringir a atividade física.

- Manter a hidratação, com preferência pela via oral; reduzir a ingestão de alimentos, e aumentar a de líquidos; se a via oral for inviável, ou a desidratação moderada ou grave, internar imediatamente e iniciar hidratação parenteral.

- Administrar loperamida ou difenoxilato judiciosamente, se o número de dejeções for elevado.

- Coletar fezes para cultura e parasitológico.

- Administrar antimicrobianos.

- Adotar medidas terapêuticas, apropriadas às manifestações sistêmicas.

\section{B- Pacientes imunocomprometidos}

Cuidados gerais, como os delineados para indivíduos previamente hígidos. 
Coletar fezes para cultura e parasitológico e obtenção de aspirado duodenal para exame microscópico, no atendimento inicial.

Considerar a possibilidade de infecção por agentes oportunistas ( Tabela III) em portadores de SIDA; o próprio HIV é causa de diarréia.

Administrar antimicrobianos e antiparasitários precocemente, em doses mais elevadas e por tempos mais longos do que habitualmente, em pacientes sem imunodepressão.

Tabela III - Agentes causadores de diarréia em pacientes imunodeprimidos

- Cryptosporidium

- Microsporidium

- Isospora belli

- Giárdia lamblia

- Vírus Epstein-Barr

- Herpes simplex

- Mycobacterium tuberculosis

- Enterocytozoon bieneusi

- Strongyloides stercoralis

- Septata intestinalis

- Entamoeba histolytica

- Citomegalovírus

- Mycobacterium avium-intracellulare

- Iatrogenia

\section{C- Diarréia nosocomial}

O Clostridium difficile é causa importante de infecção. A pesquisa das toxinas A e B do Clostridium difficile, nas fezes, deve ser feita prontamente, sempre que o paciente esteja tomando antibióticos; caso o resultado seja negativo, a retossigmoidoscopia pode revelar pseudomembranas que permitam o diagnóstico. Ocasionalmente, em hospitais, ocorrem surtos de diarréia, que podem ter como causa intoxicação alimentar, vírus ou bactérias, como Escherichia coli; pela concomitância de co-morbidades que fragilizam os pacientes, a administração precoce de antimicrobianos deve ser considerada. A Tabela IV apresenta as outras causas mais freqüentes de diarréia apresentada por pacientes hospitalizados. Em portadores de doença vascular, e, particularmente, no pós-operatório imediato de prótese aórtica, a possibilidade de colite isquêmica deve ser explorada.

\author{
Tabela IV - Causas de diarréia nosocomial \\ Íleo paralítico \\ Fecaloma ( diarréia paradoxal) \\ Dietas enterais, excessivamente concentradas \\ Infecções nosocomiais \\ Drogas \\ Colite isquêmica \\ Recidiva de condição prévia \\ Imunossupressão \\ Antibióticos
}

OLIVEIRA RB. Acute diarrhea. Medicina, Ribeirão Preto, 36: 257-260, apr./dec. 2003.

ABSTRACT - We herein present the guidelines for the approach of the patient with acute diarrhea.

UNITERMS - Diarrhea, Acute. Therapeutical Approaches.

\section{BIBLIOGRAFIA RECOMENDADA}

1 - GUERRENT RL; VAN GILDER T; STEINER TS; NATHAN M. THIELMAN NM; SLUTSKER L; TAUXE RV; HENNESSY T; GRIFFIN PM; DUPONT H; SACK RB; TARR P; NEILL M;
NACHAMKIN I; RELLER LB; OSTERHOLM MT;BENNISH ML \& PICKERING LK. Practice guidelines for the management of infectious diarrhea. Clin Infect Dis 32: 331-350, 2001.

2 - THIELMAN NM \& GUERRANT RL . Acute infectious diarrhea. $\mathbf{N}$ Engl J Med 350: 38-47, 2004. 